\title{
Design of Portable ECG Device Based on STM32
}

\author{
Luo Xiao-hui ${ }^{1,2}$ \\ 1.Chongqing College of electronic engineering, Chongqing, 401331 \\ a.luohui54@yahoo.com.cn
}

Abstract: In accordance with the big size and bulky volume and not easy to carry of ECG monito ring equipment limitations, a new portable ECG collecting equipment of real-time monitoring and low-cost was designed. Compared to the static ECG monitor , the new design has obvious advantages easy to cassy and fit to real-time operate .The device can store 24-hour ECG data and transfer data with PC through the interface of USB .The ECG waveforms can be displayed in the TFT-LCD real-time and show a good interactive interface .

Key words: ECG ; common-mode interference ; STM32 ; portable

Heart disease, as a common chronic disease it has long been a threat to human health of people. Prompting discovery and prevention are extremely important in reducing the risk of cardiovascular disease. Electrocardiogram is the main basis for the treatment of the disease. But at present, due to the limitation of the application of ECG and the price of HOLITER is very expensive. So that patients can not get real-time monitoring. The diagnosis and treatment of the patient's condition is extremely unfavorable. The emergence of portable ECG device enables ECG signal to be collected in more occasions, and it can not only realize the mobile, but also real-time ECG signal analysis. Through the built-in large capacity memory device is capable of real-time monitoring of the patient for a long time and record the patient's ECG data, and data transmission through the USB interface with PC that is to be submitted to the professional medical institutions do further analysis and diagnosis.

\section{Overview of the overall design of the system}

The schematic diagram of the system can be expressed in figure 1.ECG signal from the electrode to get, and into the ECG acquisition circuit. Through the preamplifier, main amplifier, high pass filtering, level rise are getting the required ECG signals, and sent to the ADC AD for STM32 conversion. In order to suppress the interference signal the right leg driving circuit is also introduced in the circuit. Chip of system control and use the STM32, TFT-LCD touch function plus a small button can build a good environment for human-computer interaction; can through the LCD real-time display and playback; the SD card can be stored for 24 hours ECG data; data through the USB reliable transmission to PC machine in order to make further analysis of the ECG data.

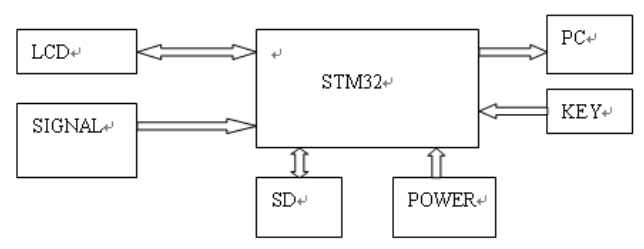

figure 1 Principle block diagram of electrocardiogram instrument

\section{System main hardware structure and circuit}

System is mainly divided into three parts: the ECG acquisition circuit is mainly to complete 
the extraction of ECG signal; bandpass filtering and main amplifier circuit, and signal collected for conditioning to meet the processing requirements; STM32 processing circuit, ECG display, analysis, and storage and data transmission functions.

\subsection{ECG acquisition circuit}

ECG acquisition circuit is the core of the whole portable ECG monitor, which directly determines the performance of the whole system. ECG acquisition circuit mainly includes: input buffer and preamplifier, right leg drive, high pass filter, main amplifier and level rise.

Body surface ECG signal frequency is mainly concentrated in $0.05 \sim 100 \mathrm{~Hz}$,for example, the amplitude is $10 \mathrm{~V} \sim 4 \mathrm{mV}$ and the typical value is $1 \mathrm{mVwhich}$ is a kind of low frequency weak bipolar signal. The ADC STM32 input voltage range is $0 \sim 3.3 \mathrm{~V}$, so the need for ECG signal amplification and level uplift, and the overall magnification of about 1000 times, and then through the level rise circuit to drive up 1V.ECG measurement and actual electrode could not completely symmetrical. This will cause the baseline drift, and ubiquitous power frequency interference $(50 \mathrm{H}$ z).EMG interference that all these require ECG preamplifier must have a high common mode rejection ratio. General requirements for common mode rejection ratio above $80 \mathrm{~dB}_{[2]}$.

This design selects INA118 instrumentation amplifier as the system preamplifier, and it has the characteristics of low noise, low drift, Gao Gongmo suppression ratio, high input impedance, its gain can be up to 1000 times, and the calculation formula is $\mathrm{G}=1+50 \mathrm{k} / \mathrm{Rg}$.Electrode polarization voltage is up to $300 \mathrm{MV}$, in order to prevent the preamplifier into cut-off or saturation state must limit the magnification. Here gain 10 , drawn by the $G=1+50 / \mathrm{Rg}$ an $\mathrm{Rg}=5.6 \mathrm{k}$, external resistor RG choose resistance 5.6k Omega precise wire wound resistor. Due to the impedance of the human body and the electrode impedance is very large, so in front of the amplifier designed to follow a signal buffer. In order to better suppress the interference of $50 \mathrm{Z} \mathrm{H}$, the right leg electrode is connected with the grounding end of the amplifier through the resistance, so as to reduce the common mode voltage of the human body[3].

\subsection{Band-pass filter and main amplifier circuit}

ECG band mainly in the $0.05 \sim 100 \mathrm{~Hz}$, so the band-pass filter design bandwidth of $0.03 \sim$ $110 \mathrm{~Hz}$ to filter out interfering signals. Band-pass filter with a low pass filter to form [4], which based on the size and cost considerations, and hardware filtering only a first-order high-pass filter and a first order low-pass filter, circuit, but there are still $50 \mathrm{~Hz}$ interference into the circuit. This is no longer designed to $50 \mathrm{~Hz}$ notch filter, but instead a software method by designing a digital filter to filter frequency interference [5] and the experimental results show that. By the low pass filtered signal waveform clear and obvious characteristics.Although some have frequency interference, it can use software designed to filter out.

\subsection{STM32 processor and main interface circuit}

STM32 series 32bit flash microcontroller using ARM company with a breakthrough of the Cortex-M 3 core, which the operating frequency of $72 \mathrm{MHz}$ and built-in high speed memory, and it is also rich enhanced I/O port and connected to the two APB bus peripherals. All models of the device are 2 ADC 12bit, 3 universal 16bit timer and a PWM timer, it also includes standard and advanced communication interface up to 2 I2C and SPI, 3 USART, one USB and one CAN. Its operating voltage is common 3.3V.The kernel is specially designed to meet the requirements of 
high performance, low power consumption, real time application and competitive price.

\section{3.1 TFT-LCD LCD interface design}

Choosing $320 * 240$ TFT liquid crystal to display the waveform, and with the FSMC STM32 module to control the liquid crystal is very suitable. FSMC is flexible static memory controller, and it can with synchronous or asynchronous memory and 16 bit PC memory card interface, and one of the major features of the is access to the timing of the external equipment can be programming: waiting period, programmable bus recovery cycle program and output enable and write enable delays programmable, and independently reading and writing timing, and protocols. In this way can you use the liquid crystal as an external storage device to use, or configured to read and write and control signal timing. As long as the specified pointer can be achieved on the liquid crystal read and write access. In this way, the first is to simplify the operation of the liquid crystal, only to specify the read and write data to complete the operation of the pointer, and the two is to improve the access speed, and avoid the use of the port to simulate the timing of the liquid crystal generated "pull screen" phenomenon.

\section{3.2 SD card interface and USB data transmission design}

SD card has the advantages of large storage capacity, low cost, fast reading and writing speed, and it is becoming the mainstream of storage devices. Its access method has two kinds: SPI mode and SDIO mode.STM32 has interface of the two modes,

ECG data storage for the portable ECG device is necessary, and this paper in the storage design realizes two functions: one is to support 24-hour ECG data storage; the second is established based on the SD card file system; the ECG data is stored as TXT file format. This process has an advantage, both the USB interface with the PC machine can be used for data transmission, and the SD card can be pulled down with a card reader to read data into the PC machine. STM 32 contains USB module, so eliminating the need to expand the USB chip, and the other ST also provides a large number of USB examples, only a little modification can be applied to the actual project, speeding up the development process.

\section{Software design}

Software uses the function modular design method, through the analysis, it can obtain the control system main program and the ADC interrupt procedure software flow chart. The main program mainly completes the graph menu and the wave shape drawing. ADC interrupt service routine to complete the acquisition of voltage and the transfer of the flag bit.

\section{Test result analysis}

One end of the three electrodes are respectively connected with the left arm and the right leg of the human body, so that one end of the three input end is connected with the input ends of the acquisition circuit, and the waveform of the sampling frequency $200 \mathrm{~Hz}$ is shown in Figure 2. 


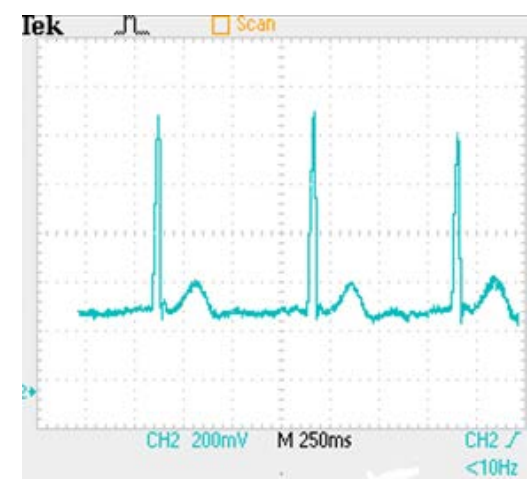

figure 2 The waveform of the liquid crystal display at the sampling frequency of $200 \mathrm{~Hz}$ TFT liquid crystal display from Figure 2 shows that the waveform of the ECG display waveform is clear and stable, and the waveform characteristics are obvious, and can meet the needs of practical applications.

\section{Summary}

STM32 as the main control chip, which contains a wealth of functional modules. With standard and advanced communication interface, and without the need to expand the chip to complete the acquisition, and storage and data communication. The whole ECG instrument has the characteristics of small size and low power consumption. Meet the basic requirements of portable equipment. The experimental results show that the system achieves the expected effect. With the increasing incidence of cardiovascular diseases, the portable ECG instrument has high application value and good market prospect.

\section{References}

[1] Dai Shaosheng, Zhang Yuesun, Zhang Hejun, et al. A portable remote real-time ECG monitor. System [J]. Medical and health equipment, 2006, 27 (9): 1-2.

[2] Huang Minsong, Hong Yan, Liu Jiancheng.ECG preamplifier circuit design.Electronic technology.2007, 16: 187-189.

[3] Wint er $\mathrm{B} B$, Webs ter $\mathrm{J} G$.Dri ven-right-leg $\mathrm{Ci}$ rcuit De-sig $\mathrm{n}$.IEEE $\mathrm{T}$ rans .Biomed .Eng .1983, BME-30 :62-66.

[4] Tong Sibai. Analog electronic technology [M]. Beijing: higher education press,2001.133-148.

[5] Xu Sheng et al. Modern digital signal processing [M]. Beijing: Mechanical EngineeringIndustry Press, 2005.129-148. 